\title{
CALCULATION OF CROSS SECTIONS AND ASTROPHYSICAL S-FACTORS AT 0-5 MeV ENERGY RANGE FOR ${ }^{8} \mathrm{Li}(\alpha, n){ }^{11}$ B REACTION
}

\author{
Ercan YILDIZ 1,*, İsmail Hakkı SARPÜN ${ }^{2,3}$ \\ ${ }^{1}$ Kahramanmaras Sutcu Imam University, Vocational School of Health Services, Department of Medical Imaging \\ Techniques, Kahramanmaras, Turkey \\ ${ }^{2}$ Akdeniz University, Physics Department, Antalya, Turkey \\ ${ }^{3}$ Akdeniz University, Nuclear Sciences Research and Application Center, Antalya, Turkey
}

\begin{abstract}
Explaining the origin and evolution of elements is one of the most important problems in astrophysics. The r-process in the current nucleosynthesis scenario; roughly responsible for the production of half of the nuclei heavier than iron. ${ }^{8} \operatorname{Li}(\alpha, n){ }^{11} \mathrm{~B}$ reaction plays an important role in the r-process of this scenario because of being a cosmic radiation induced reaction. In particular, it is important to know the change with energy in the low energy zone for testing the accuracy of the model calculations. In this study, the astrophysical S-factors of the ${ }^{8} \operatorname{Li}(\alpha, n){ }^{11} \mathrm{~B}$ reaction and the probability of reaction at low energies were calculated in the 0-5 MeV energy range. Since the determination of fusion cross section at very low energy becomes a critical issue with the lacking of data from the experimental measurements, theoretical calculation of the astrophysical S-factor can be substantial. It is thought that the lack of measurement in the low energy region will be eliminated by the astrophysical S-factor. TALYS 1.8 code was used for the theoretical calculations. The obtained theoretical values were compared with experimental data obtained from the EXFOR database.
\end{abstract}

Keywords: ${ }^{8} \mathrm{Li}$, Cross-Section, S-Factor, TALYS 1.8

\section{INTRODUCTION}

The question of how the universe came into being always stands before us as a question that scientists always wonder and develop various theories. Studies on this subject produced new questions especially when trying to explain the formation of the universe. Researching the origin and evolution of chemical elements is one of the most important subjects of astrophysics studies. Significant concordance between the basic nucleosynthesis calculations and the observed abundance of light nuclides $\left({ }^{2} \mathrm{H},{ }^{3} \mathrm{He},{ }^{4} \mathrm{He}\right.$ and ${ }^{7} \mathrm{Li}$ ) are counted among the achievements of the standard Big Bang model [1].

The elements with an atomic number up to iron, which are considered as lighter, are produced by nuclear combustion reactions in stars, while the elements which atomic numbers greater than iron, considered as heavy, are produced in explosion environments, such as supernova. The stability curve is an important line in the graph of proton number versus neutron number of nuclei. The nuclei in the neutron-rich part of the stability curve in the nucleus table are produced by the s- and r-processes, while the nuclei in the proton-rich part are produced by the p-process.

In the present nucleosynthesis process, the r-process still has nuclear astrophysical aspects still waiting to be discovered, although it is responsible for the production of roughly half of the heavy nuclei [2]. Reactions $(\alpha, n)$ and $(p, n)$ on light and neutron-rich radioactive nuclei play important roles in the onset of the r-process nucleosynthesis, for example in a neutron-rich environment. Although ${ }^{8} \operatorname{Li}(\alpha, n){ }^{11} \mathrm{~B}$ reaction is important for predicting the abundance of elements heavier than $\mathrm{Li}$ in the early universe, ${ }^{8} \mathrm{Li}(\alpha, n){ }^{11} \mathrm{~B}$ reaction $\mathrm{A}=8$ stable state in neutron rich environment (temperature dependent normalized distribution function at $\mathrm{T}_{9}=1$ ). It is one of the key reactions for the formation of increasingly heavier elements throughout the formation of a steady state (at the temperature of billions of Kelvin) [3]. The

*Corresponding Author: ercan7306@hotmail.com

Received: 11.10.2019 Published: 31.03.2020 
measurement of reaction cross-sections, which is a general problem in nuclear astrophysics, also occurs for the ${ }^{8} \mathrm{Li}(\alpha, \mathrm{n}){ }^{11} \mathrm{~B}$ reaction. The cross-sections of Gamow energy $(0.5$ to $2 \mathrm{MeV}$ for this reaction) are too small to be measured in the laboratory [4]. Cross-section calculations for ${ }^{8} \mathrm{Li}(\alpha, n){ }^{11} \mathrm{~B}$ reaction are prediction studies using several statistical models rather than experimental studies [1]. Similarly, direct measurement of neutron capture reactions from light nuclei, especially for radioactive nuclei, such as ${ }^{8} \mathrm{Li}$ and ${ }^{8} \mathrm{~B}$, is impossible, and indirect calculation methods have been applied to overcome these difficulties [5]. In the early stages of the formation of the universe, the evolution of stars, nova, supernova explosion, X-ray explosions and so on. In the analysis of many astrophysical phenomena, the important input parameter in the models is the effect cross-section values of the reactions. However, since the temperature is not usually very high in these environments, the cross-section values at low energies, ie, the cross-sections of the "Gamow Barrier" energies, which are considered the beginning of the Coulomb barrier, must be obtained [5].

For some reactions, that cross-sections cannot be measured directly with low energies, some procedures or various methods are used to obtain cross-sections in this region. The simplest way to obtain crosssectional values is to approach low energy values by means of extrapolation. The effect cross-sections of charged particle interactions can be obtained in the low energy region from the S-factor values obtained by extrapolation from the S-factor values calculated from the current cross-section values in the high energy region [5].

The astrophysical S-factor is related to the cross-sections $\sigma(\mathrm{E})$ in the field dimension and $\mathrm{E}$ energy value, which is the main character of a reaction. The cross-sections of the reactions of charged particles in stars are governed by Coulomb effects [6].

$$
\begin{gathered}
S(E)=\sigma(E) E \exp (2 \pi \eta) \\
\sigma(E)=E^{-1} S(E) \exp (-2 \pi \eta)
\end{gathered}
$$

Here, $\eta$ is the Sommerfeld parameter and is expressed by $\left(Z_{1} Z_{2} e^{2}\right) / \hbar v . Z_{l}$ and $Z_{2}$ are the loads of the $1 \mathrm{st}$ and 2 nd cores that interact. The energy-dependent $S(E)$ function represents nuclear interactions within the cross-section. This function is called an astrophysical S-factor, and in the absence of resonance, its dependence on energy is very low. Since the energy-dependent variation of the astrophysical S-factor is much slower than the energy-dependent change of the cross-section, it is much more convenient to extrapolate the astrophysical S-factor to lower energies where experimental cross-section measurement is not possible. In order to test the accuracy of model calculations, it is especially important to know the change in low energies [4].

In this study, we aimed to investigate the astrophysical S-factor change for the ${ }^{8} \operatorname{Li}(\alpha, n){ }^{11} \mathrm{~B}$ reaction in the low energy region, ie in the $0-5 \mathrm{MeV}$ energy range.

\section{EXPERIMENTAL DETAIL}

In this study, reaction effect sections of ${ }^{8} \mathrm{Li}(\alpha, n){ }^{11} \mathrm{~B}$ reactions and astrophysical S-factors were calculated using these values. Therefore, $(\alpha, n)$ reaction sections were first calculated using the TALYS 1.8 [7] nuclear reaction code in the energy range between 0 and $5 \mathrm{MeV}$. Then, the effect cross-sectional values and (1) equation were used to obtain astrophysical S-factor values.

TALYS 1.8 [7] nuclear code program; photon, neutron, proton, deuteron, triton, helium and alpha particles formed by the target core mass is 7 and heavier, provided that the nuclear reaction code is used to study the nuclear reactions. On the other hand, in order to make comparisons between experimental and calculated data, the relevant experimental values were taken from EXFOR [8] (International Experimental Nuclear Data Library). 


\section{RESULTS and DISCUSSION}

Especially in the low energy region, ie $0-5 \mathrm{MeV}$ range ${ }^{8} \mathrm{Li}(\alpha, n){ }^{11} \mathrm{~B}$ for the reaction with the TALYS 1.8 nuclear code obtained with the theoretical effect cross-section obtained by comparing the experimental $[1,9,10]$ values obtained by comparing Figure 1 is also given. The comparison of the theoretical astrophysical S-factor values calculated using the cross-sections obtained from TALYS 1.8 [7] with experimental values is given in Figure 2.

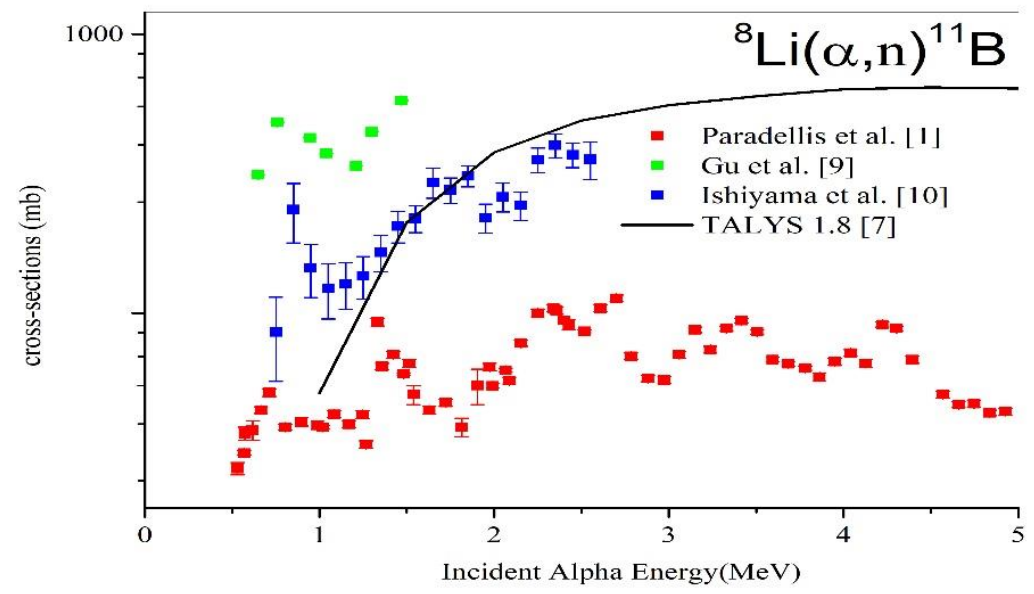

Figure 1. Experimental and theoretical cross sections for ${ }^{8} \mathrm{Li}(\alpha, \mathrm{n}){ }^{11} \mathrm{~B}$ reaction

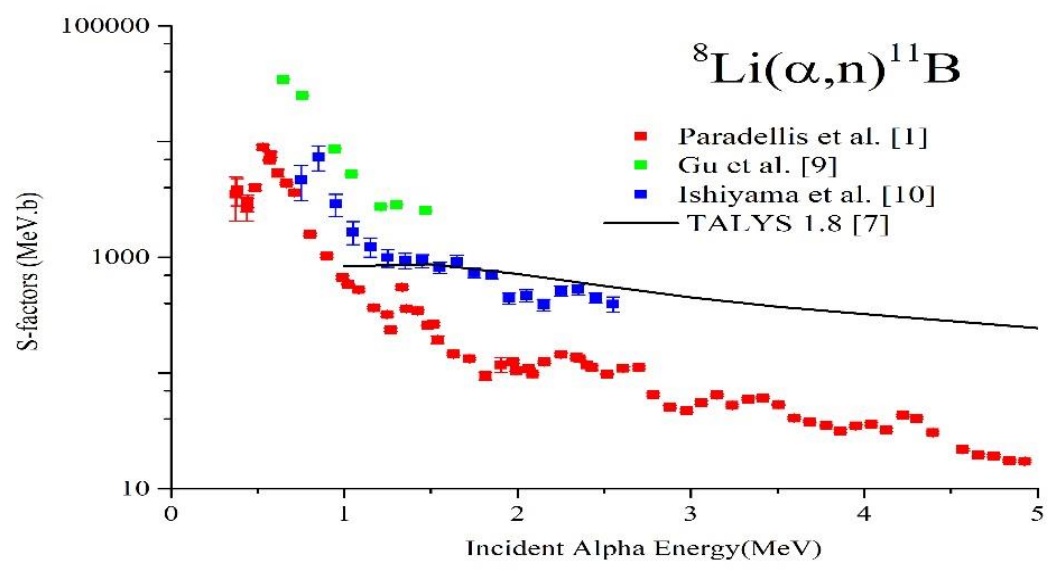

Figure 2. Experimental and theoretical astrophysical $S$-factor values for ${ }^{8} \mathrm{Li}(\alpha, \mathrm{n}){ }^{11} \mathrm{~B}$ reaction

Many alpha-induced reactions using the TALYS 1.8 [7] nuclear code have been theoretically calculated and shown to yield accurate results [11-13]. Similarly, although the cross-sections obtained for the ${ }^{8} \mathrm{Li}(\alpha, \mathrm{n}){ }^{11} \mathrm{~B}$ reaction were in the low energy region, Ishiyama et al. [10] are in good agreement with the experimental values.

When we examined the experimental and theoretical astrophysical $S$-factor values for the ${ }^{8} \operatorname{Li}(\alpha, n){ }^{11} B$ reaction in Figure 2, Ishiyama et al. [10] and TALYS 1.8 [7]. In addition, in the two experimental data groups obtained from EXFOR [8] in Figure 2, Ishiyama et al. [10], Paradellis et al. [1] and Gu et al. It can be seen that [9] values show parallelism in the range of 1-2 MeV. If the experimental and theoretical astrophysical S-factor values for the ${ }^{8} \mathrm{Li}(\alpha, n){ }^{11} \mathrm{~B}$ reaction in Fig. 2 are examined, TALYS 1.8 [7] data 
values of $\mathrm{Gu}$ et al [9]. It can be stated that it is incompatible with [9] values. Paradellis et al. [1] experimental values, especially around the Gamow peak region.

\section{CONCLUSIONS}

S-factors are widely used in astrophysics applications for extrapolations to the very low energies of the astrophysically relevant Gamow energy region. Particularly with the semi-empirical formulas to be obtained for the S-factors it will be possible to obtain cross-sectional values for the low energy region. This allows comparison of experimental and nuclear model calculations. In this way, more up-to-date data can be obtained.

The lack of data is remarkable in the low-energy region and the Gamow energy region of the ${ }^{8} \operatorname{Li}(\alpha, n){ }^{11} \mathrm{~B}$ reaction, which is an important step in the production of neutron-rich nuclei.

For a better theoretical approach and contribution to astrophysical systems, new measurements are needed, particularly for energies below $1 \mathrm{MeV}$. Therefore, theoretical calculations can also be repeated with new nuclear parameters to obtain the best fit with the experimental data. The neutron-rich nuclei produced by the s- and r-processes in the isotope table will contribute to the physical structure and classification of the isotopes found in the proton-rich part produced by the p-process.

In particular, it will be possible to obtain cross-sectional values using the semi-empirical formulas for the $\mathrm{S}$ factor and Eq. (2) for the low energy region.

It is thought that the abundance of the elements in the solar system will be a step for understanding the formation mechanisms of these processes at different time intervals and the processes of different temperature and neutron densities.

The question of how the universe came into being always stands before us as a question that scientists always wonder and develop various theories. Studies on this subject produced new questions especially when trying to explain the formation of the universe.

It is thought that our study will generate new questions and provide a good reference for future crosssection studies and nuclear model calculations.

\section{REFERENCES}

[1] Paradellis T, Kossionides S, Doukellis G, Aslanoglou A, Assimakopouls P, Pakou A, Rolfs C and Langanke K. Astrophysical $\mathrm{S}(\mathrm{E})$ factor of ${ }^{8} \mathrm{Li}\left(\alpha, \mathrm{n}_{0}\right)^{11} \mathrm{~B}$ and inhomogeneous Big Bang nucleosynthesis, Zeitschrift für Physik A Atomic Nuclei, 1990; 337: 211-220.

[2] Terasawa M, Sumiyoshi K, Kajino T, Mathews GJ and Tanihata I. New Nuclear Reaction Flow during r-Process Nucleosynthesis in Supernovae: Critical Role of Light, Neutron-rich Nuclei, The Astrophysical Journal, 2001; 562: 470-479.

[3] Miyatake H, Ishiyama H, Tanakaa MH, Watanabea YX, Yoshikawa N, Jeonga SC, Matsuyama Y, Fuchia Y, Nomuraa T and Hashimotoh T. Exclusive measurement of the astrophysical ${ }^{8} \operatorname{Li}(\mathrm{a}, \mathrm{n})$ reaction cross section, Nuclear Physics A, 2004; 738: 401-405.

[4] Yıldız E, 0-8 MeV Enerji Bölgesinde ${ }^{13} \mathrm{C}(\alpha, n){ }^{16} \mathrm{O}$ Reaksiyonu için Astrofiziksel S-Faktörlerin Hesaplanmas1, Süleyman Demirel Üniversitesi Fen Edebiyat Fakültesi Fen Dergisi, 2018; 13: 2528. 
[5] Guimarâes V and Bertulani CA. Light radioactive nuclei capture reactions with phenomenological potential models, AIP Conference Proceedings, 2010; 30: 1245.

[6] Alinka LS and Descouvermont P. Nuclear astrophysics: Nucleosynthesis in the Universe International, Journal of Astrobiology, 2012, 11: 243-250.

[7] Koning AJ, Hilaire S and Goriely S. TALYS-1.8 A Nuclear Reaction Program, 2016.

[8] EXFOR/CSISRS, Brookhaven National Laboratory, National Nuclear Data Center, Database Version of September 2017.

[9] Gu X, Boyd RN, Farrel MM, Kalen JD, Mitchell CA, Kolata JJ, Belbot M, Lamkin K, Ashktorab $\mathrm{K}$ and Bechetti FD. The ${ }^{8} \mathrm{Li}(\alpha, n){ }^{11} \mathrm{~B}$ reaction and primordial nucleosynthesis, Physics Letter B, 1995; 343: 31-35.

[10] Ishiyama H, Hashimoto T, Ishikawa T, Watanabe YX, Das SK, Miyatakea H, Mizoi Y, Fukuda T, Tanaka MH and Fuchi Y. A new measurement of the astrophysical ${ }^{8} \mathrm{Li}(\alpha, n)$ reaction, Physics Letter B, 2006; 640: 82-85.

[11] Kaplan A, Investigation of Neutron-Production Cross Sections of the Structural Fusion Material ${ }^{181} \mathrm{Ta}$ for $(\alpha, \mathrm{xn})$ Reactions up to $150 \mathrm{MeV}$ Energy, Journal of Fusion Energy, 2013; 32: 382-388.

[12] Sarpün İH, Aydın A, Kaplan A, Koca H and Tel E, Comparison of Fission Barrier and Level Density Models in (a,f) Reactions of Some Heavy Nuclei, Ann. Nucl. Energy 2014; 70: 175-179.

[13] Aydın A, Tel E, Pekdoğan H, and Kaplan A, Nuclear Model Calculations on the Production of ${ }^{125,123} \mathrm{Xe}$ and ${ }^{133,131,129,128}$ Ba Radioisotopes, Phys. Atom Nucl. 2012; 75: (3), 310-314. 